\title{
Acute conjunctivitis and corneal foreign bodies secondary to tarantula hairs
}

\author{
Yelin Yang BHSc MD, Ted Christakis BSc MD, Kamiar Mireskandari MBchB PhD
}

Competing interests: None declared.

This article has been peer reviewed.

The authors have obtained patient consent.

Correspondence to: Kamiar Mireskandari, kamiar.mireskandari@ sickkids.ca

CMAJ 2016. DOI:10.1503 /cmaj.150571
1 n otherwise healthy three-year-old boy was referred to the emergency department with a one-day history of redness, pain and sensation of foreign bodies in his left eye. His parents had held a birthday party the day before, during which he was in contact with exotic animals, including a tarantula. No other children at the party were symptomatic. Treatment with erythromycin ointment four times daily was prescribed by the referring physician for possible conjunctivitis.

On examination (day 3), the patient's visual acuity was $20 / 25(6 / 7.5)$ in each eye. His parents recalled seeing their child start blinking, rubbing his eyes and crying within seconds after holding a Chilean rose tarantula (Figure 1). The ophthalmology service was consulted, and slit-lamp examination showed substantial conjunctival injection, chemosis and numerous intracorneal linear foreign bodies in the left eye (Figure 2A and B). The area primarily affected was the anterior portion of the corneal stroma. The anterior chamber showed no inflammatory reaction. Findings on examination of the posterior segment were unremarkable. The fine barbed hairs from the tarantula were embedded in the cornea and covered by the overlying epithelium of the cornea (no fluorescein staining). We did not attempt to remove the hairs, because it would have required general anesthesia and likely would have led to more localized scarring from trauma associated with the procedure. Treatment with the erythromycin ointment was continued for two weeks, and the patient was given an eye shield to wear to prevent rubbing.

On day 32, the patient was symptomatically better, with few episodes of foreign-body sensation and eye redness, and minimal photosensitivity. His vision improved to 20/20 (6/6) in each eye. The tarantula hairs had migrated deeper into the cornea, with increased surroundin $\mathrm{g}$ stromal haze (Figure 2C). By day 66, a few hairs were found protruding into the anterior chamber, with local reaction. Encapsulation was seen on day 82. Encapsulated barbs were visible at one-year follow-up (Figure 2D). No inflammation of the anterior chamber was noted at any stage of follow-up. At two years following the injury, the patient was asymptomatic, with no further changes in his ocular examination. The corneal endothelial cell density, an indicator of corneal health measured by specular microscopy, did not differ between the eyes (3096 cells $/ \mathrm{mm}^{2}$ in the right eye and 3058 cells $/ \mathrm{mm}^{2}$ in the left eye).

\section{Discussion}

Tarantulas are members of the Theraphosidae family of large hairy spiders, with a life expectancy of up to 20 years. They have become a popular choice of pet because they require little maintenance, are attention-grabbing and are widely available. In 2008, $21 \%$ of Canadian households were estimated to have exotic pets,

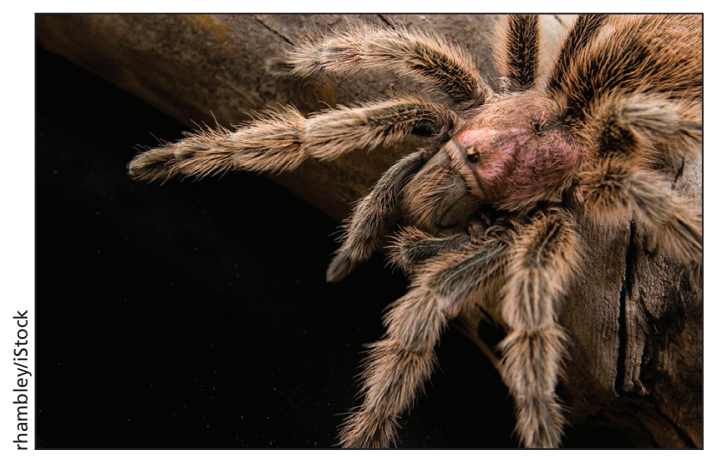

Figure 1: Image of a Chilean rose tarantula. 
including tarantulas, fish and reptiles. ${ }^{1}$ Although tarantula bites are venomous, the Chilean rose tarantula is relatively harmless. That said, health risks related to their defensive, barbed hairs, or setae, are not well recognized. These fine, urticating hairs are densely packed on the tarantula's abdomen (10 000 per $\left.\mathrm{mm}^{2}\right)$ and form part of the spider's defence mechanism. ${ }^{2}$ They are released by being sprayed into the air or through direct contact. ${ }^{2}$ There are four types of urticating hairs in tarantulas, categorized by the pattern of barbs. ${ }^{3}$ A species can have more than one type. However, type III hairs are the most clinically significant, because they are able to penetrate the cornea deeply and cause irritation. They are morphologically similar to caterpillar hairs, which are long, thin, pointed and barbed (Figure 3$){ }^{2}$ These features facilitate deep tissue penetration, which causes skin, respiratory and ocular irritations. A granulomatous nodular reaction of the conjunctiva and iris has long been described in relation to insect and even plant hairs. ${ }^{4}$

\section{Clinical presentation and course}

Patients typically present with ocular irritation, tearing, eye redness and surrounding skin irritation. Linear corneal abrasions may be seen on examination, which suggests the presence of foreign bodies. ${ }^{4,5}$ The effect of urticating hairs on the eye is related to their mechanical penetration and direct toxicity. ${ }^{5}$ The hairs can lodge in any ocular tissue, most commonly the palpebral conjunctiva, followed by the cornea, anterior chamber, vitreous and retina. ${ }^{5}$ Clinical manifestations range from mild keratoconjunctivitis, granulomas and iritis to involvement of deeper intraocular structures, including papillitis and chorioretinitis. ${ }^{5}$ In a small proportion of patients with intraocular penetration, serious conse-

\section{Box 1: Classification of ocular pathologies based on exposure to caterpillar hairs ${ }^{6}$}

Type 1: Acute toxic reaction after exposure to hairs lasting days; marked by chemosis, foreignbody sensation, tearing

Type 2: Chronic keratoconjunctivitis from hairs lodged in bulbar or palpebral conjunctiva; may have corneal abrasions

Type 3: Granuloma formation (hairs migrate under conjunctiva and to cornea, leading to localized inflammation)

Type 4: Iritis from hairs entering anterior chamber; may have iris nodules and intralenticular foreign bodies

Type 5: Vitreoretinal involvement from hairs penetrating into posterior segment quences can develop that result in long-term visual impairment. The spectrum of ocular pathologies has been classified into five types based on exposure to caterpillar hairs (Box 1), ${ }^{6}$ which causes more severe disease but may be extrapolated to tarantula hairs. Most cases are type 1 or 2 , involving acute toxic reaction to hairs or keratoconjunctivitis.

Once lodged into the cornea, the hairs tend to migrate deeper over time. This is likely secondary to the movement of the globe or production of inflammatory exudate propelling the hairs forward. ${ }^{7}$ Eye rubbing may exacerbate this process. In a retrospective chart review of caterpillarinduced ophthalmitis over the course of three years, $14.7 \%(80 / 544)$ of patients had intracorneal hairs, and intraocular migration was noted in $24 \%(19 / 80)$ of them. ${ }^{5}$ The hairs are usually reabsorbed without complications. However,
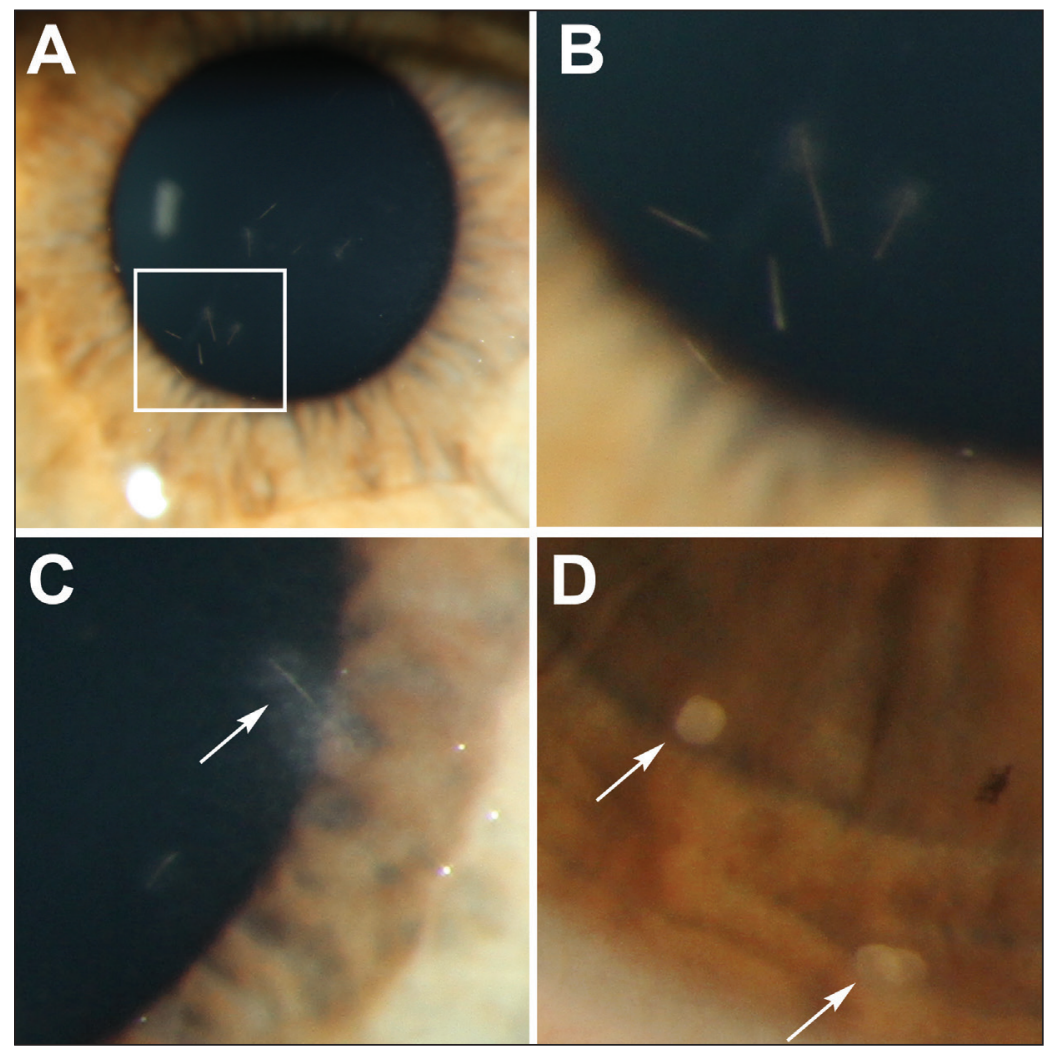

Figure 2: Slit-lamp microscopy, showing intracorneal tarantula hairs in the left eye of a three-year-old boy on day 3 after holding a Chilean rose tarantula (A and B [magnification]); hairs migrating deeper into the cornea, with increased surrounding stromal haze, on day 33 (C); and encapsulated hair barbs visible at one-year follow-up (D).

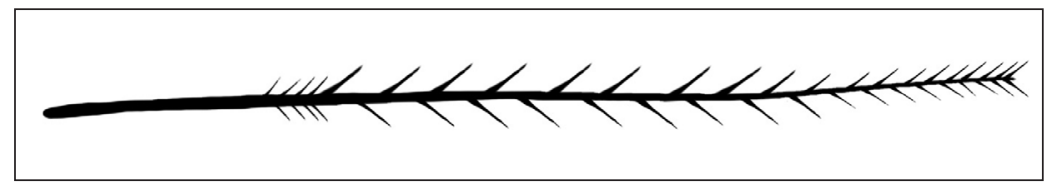

Figure 3: An example of a type III urticating hair found on caterpillars and tarantulas. They are long, thin, pointed and barbed - features that facilitate deep tissue penetration. 
cases of panuveitis have been reported, with a long-term impact on vision. In one patient, despite attempts at hair removal and complete resolution of symptoms, the remaining hairs migrated to the posterior segment, causing panuveitis one year after the initial injury. ${ }^{8}$

In our case, few hairs progressed to protrude into the anterior chamber three months after the incident. Luckily our patient did not have involvement of deeper intraocular structures. Nonetheless, the risk of intraocular migration and complications reinforces the importance of careful long-term follow-up.

\section{Management}

The treatment of embedded hairs depends on the location and depth of the hairs. Complete removal of visible hairs should be attempted, with possible sedation in children. However, this is often difficult because of the friability of hairs, barbs hooking into the corneal stroma and poor visibility within the edematous cornea. ${ }^{9}$ The light brown colour of hairs found on Chilean rose tarantulas presents additional challenges to removal. ${ }^{9}$

For deeper intracorneal hairs, conservative management with careful observation is recommended. ${ }^{9}$ Topical steroids may be required to suppress inflammation; however, local adverse events such as glaucoma and cataract can be problematic. More invasive treatments such as laser photocoagulation and intraocular surgery to remove offending hairs have been described., ${ }^{4,9}$ However, literature on the outcome of these interventions is limited, with one report to date using argon laser photocoagulation to treat endophthalmitis from vitreous hairs. ${ }^{10}$ The laser works by fragmenting the hairs so that they lose their barbed characteristic and hence stop penetrating deeper. However, this disruption causes local inflammation, and its effectiveness has not been established. Given the potential for complications, including chronic low-grade uveitis and secondary glaucoma even in the quiescent stage of disease, long-term follow-up is required until results from ocular examinations are stabilized.

\section{Conclusion}

Although tarantulas are a relatively popular pet, the ocular complications are not well recognized among pet owners and organizers of exotic animal parties. This, along with the difficulty in detecting the fine hairs and the clinical similarity to more common causes of red eye such as infective conjunctivitis, often leads to a delayed diagnosis. Tarantula owners and people attending exotic animal parties need to be made aware of the ocular risks related to handling these animals. Precautions include wearing gloves and goggles, hand washing and avoiding eye rubbing. Any patients with a red, painful eye and a history of handling tarantulas or other hairy insects require prompt referral to an ophthalmologist and long, careful follow-up.

\section{References}

1. Canadian exotic pet ownership statistics. Ottawa: Pet Industry Joint Advisory council; 2008 [updated]. Available: www.pijaccanada. com/en/commonDocs/Membership/IndustryStats/Canadian\%20 Exotic\%20Pet\%20Ownership\%202008.pdf (accessed 2015 June 15).

2. Battisti A, Holm G, Fagrell B, et al. Urticating hairs in arthropods: their nature and medical significance. Annu Rev Entomol 2011;56:203-20.

3. Cooke JA, Miller FH, Grover RW, et al. Urticaria caused by tarantula hairs. Am J Trop Med Hyg 1973;22:130-3.

4. Bernardino CR, Rapuano C. Ophthalmia nodosa caused by casual handling of a tarantula. CLAO J 2000;26:111-2.

5. Sengupta S, Reddy PR, Gyatsho J, et al. Risk factors for intraocular penetration of caterpillar hair in ophthalmia nodosa: a retrospective analysis. Indian J Ophthalmol 2010;58:540-3.

6. Cadera W, Pachtman MA, Fountain JA, et al. Ocular lesions caused by caterpillar hairs (ophthalmia nodosa). Can J Ophthalmol 1984;19:40-4.

7. Sridhar MS, Ramakrishnan M. Ocular lesions caused by caterpillar hairs. Eye (Lond) 2004;18:540-3.

8. Hom-Choudhury A, Koukkoulli A, Norris JH, et al. A hairy affair: tarantula setae-induced panuveitis requiring pars plana vitrectomy. Int Ophthalmol 2012;32:161-3.

9. Watts P, Mcpherson R, Hawksworth NR. Tarantula keratouveitis. Cornea 2000;19:393-4.

10. Marti-Huguet T, Pujol O, Cabiro I, et al. Endophthalmos caused by intravitreal caterpillar hairs: treatment by direct photocoagulation with argon laser. J Fr Ophtalmol 1987;10:559-64.

Affiliations: Faculty of Medicine (Yang), University of Ottawa, Ottawa, Ont.; Department of Ophthalmology and Visual Sciences (Christakis, Mireskandari), University of Toronto, The Hospital for Sick Children, Toronto, Ont.

Contributors: Yelin Yang and Ted Christakis drafted the manuscript; Kamiar Mireskandari oversaw and revised the manuscript. All of the authors approved the final version to be published and agreed to be guarantors of the work.

Acknowledgement: The authors thank Cynthia VandenHoven, medical imaging specialist in the Department of Ophthalmology and Visual Sciences, University of Toronto and The Hospital for Sick Children, for her assistance with the clinical photographs.

The section Cases presents brief case reports that convey clear, practical lessons. Preference is given to common presentations of important rare conditions, and important unusual presentations of common problems. Articles start with a case presentation (500 words maximum), and a discussion of the underlying condition follows (1000 words maximum). Visual elements (e.g., tables of the differential diagnosis, clinical features or diagnostic approach) are encouraged. Consent from patients for publication of their story is a necessity. See information for authors at www.cmaj.ca. 